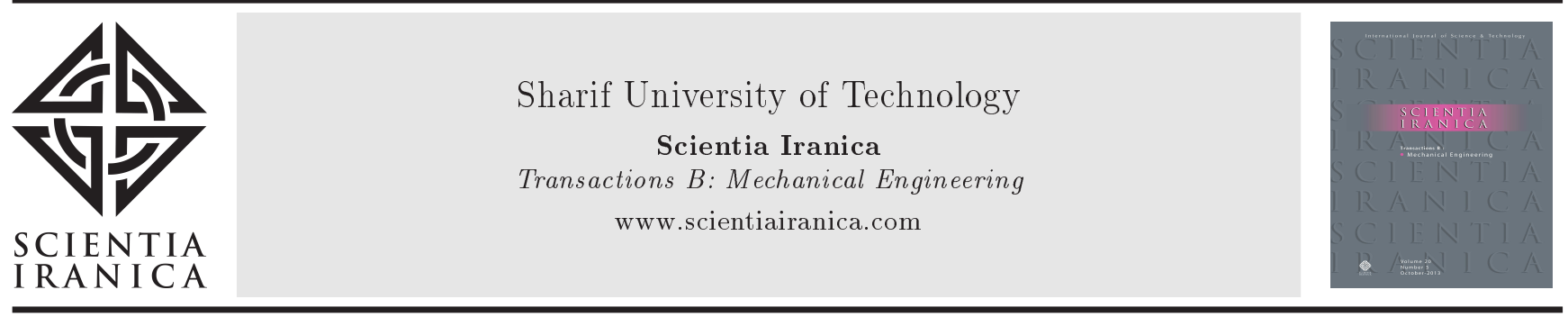

Research Note

\title{
Robust decentralized control of consensus-based formations of leader-follower networks with uncertain directed topologies on bounded velocity trajectories undefined for followers via backstepping method
}

\author{
H. Sayyaadi* and A. Soltani \\ School of Mechanical Engineering, Sharif University of Technology, Tehran, P.O. Box 11155-9567, Iran. \\ Received 4 May 2014; received in revised form 14 December 2014; accepted 1 August 2015
}

\author{
KEYWORDS \\ Decentralized control; \\ Consensus; \\ Formation; \\ Leader-follower; \\ Directed topologies; \\ Backstepping.
}

\begin{abstract}
In this paper, decentralized control of formation of a special category of leaderfollower networks on bounded velocity trajectories is addressed. The network of the agents in this study is supposed to have a directed graph with a spanning tree rooted at the leader agent. Moreover, follower agents do not receive online or have offline velocity of the desired trajectory, such as in tracking problem of trajectories which are not predefined or when the total bandwidth is narrow. Furthermore, the leader does not receive any information from any agent and its control is fully centralized. In the present study, formation problem is considered a consensus problem. The controller is designed for integrator and doubleintegrator agents via backstepping. Furthermore, suitable condition of the robustness of the controller against the changes of the communication topology of the network is derived. Simulations verify the capability and robustness of the designed control law. In a simulation, formation keeping error reduction by tuning a gain of the controller, as claimed in the design procedure, is demonstrated.
\end{abstract}

(C) 2016 Sharif University of Technology. All rights reserved.

\section{Introduction}

In the recent decade, due to advancements in designing and producing high speed processors and wireless networks developments, researchers have been interested in performing complicated tasks by multiagent systems of ordinary robots instead of designing super-mature robots. Therefore, various missions have been defined for multi-agent systems such as flocking [1], coverage [2], rendezvous [3], deployment [4],

* Corresponding author. Tel.: +98 2166165682 ; Fax: +982166000021

E-mail addresses: sayyaadi@sharif.edu (H. Sayyaadi); asoltani@mech.sharif.edu (A. Soltani) formation [5], and, in a more general case, consensus [6,7]. Due to high bandwidth necessary for centralized controllers and failure of whole mission due to failure of a single-agent in centralized missions, decentralized control laws are preferred apparently. Formation methods, till now, can be classified to three approaches, including: behavior-based approach [8], virtual structure approach [9], and Leader-follower approach [10]. Robustness of the proposed formation algorithms against changes in communication topology, which are probable as a result of surrounded environmental terms and conditions, is so important. For example, in [11], a robust decentralized attitude formation controller against information flow changes and disturbances is designed via sliding mode method. 
In [12], by a method named leader-to-formation stability method, interconnection topology is correlated to stability and ways of improving robustness of formation in spite of changes of information flow topology have been suggested. In [13], suitable condition for robustness of formation of multi-agent systems in case of time varying communication graphs has been addressed.

The present paper deals with decentralized control of formation of leader-follower multi-agent system with directed networks having spanning tree on bounded velocity trajectories. The leader of the networks considered in this study is supposed not to be receiver of information from the followers. On the other hand, the followers do not know or receive any explicit information about the desired trajectory.

The first section of the present paper is about some of the needed preliminaries and theorems and lemmas about the networks. Control design section introduces the general properties of the considered category of networks in this study. So, control laws for integrator and double integrator agents are designed via backstepping method. Furthermore, robustness condition of the controller on the networks with uncertain topology of communications is derived. Finally, some simulations are performed to exhibit the capability, limitations, and robustness of the designed control law.

\section{Preliminaries}

For an index set $I=\{1,2, \cdots, n\}$, a digraph $G$ consists of a triple $(V, \varepsilon, A)$ in which $V=\left\{v_{i} \mid i \in I\right\}$ is a finite nonempty set of nodes and $\varepsilon=\left\{e_{i j}=\left(v_{i}, v_{j}\right) \mid i, j \in I\right\}$ is the edge set and $A=\left\{a_{i j} \mid a_{i j} \neq 0 \Leftrightarrow e_{j i} \in \varepsilon, a_{i j}=\right.$ $\left.0 \Leftrightarrow e_{j i} \notin \varepsilon\right\}$ is the adjacency matrix. $v_{i}$ and $v_{j}$ are called tail and head of the edge $\left(v_{i}, v_{j}\right)$. It is assumed that $a_{i i}=0$ and $\forall a_{i j} \geq 0, i \neq j$. The adjacency matrix can be weighted or unweighted. The Laplacian matrix associated with graph $G$ is defined as $L=\Delta-A$ in which $\Delta_{i i}=\operatorname{deg}_{i n}\left(v_{i}\right)$ and $\Delta_{i j}=0$ for all $i \neq j$ and $\operatorname{deg}_{i n}\left(v_{i}\right)=\sum_{j=1}^{n} a_{i j}$. The Laplacian matrix always has a zero eigenvalue with the right eigenvector of one, because the sum of its columns is zero and so its determinant is zero, which means that the matrix has a zero eigenvalue with right eigenvector of one. These eigenvalue and eigenvector are denoted by $\lambda_{1}=0, w_{r}=1=[1,1, \cdots, 1]^{T}$. Moreover, it should be noted that a digraph has spanning tree if there is at least one node (named root) that can reach all the other nodes following the direction of information flow. In leader-follower multi-agent systems, the root of spanning tree is usually set at the leader node. A useful theorem about digraphs with spanning tree is expressed as follows.

\section{Theorem}

If a digraph has a spanning tree, then its Laplacian matrix has a simple zero eigenvalue associated with an eigenvector 1 and all of the other eigenvalues have positive real parts [14].

Laplacian matrix is used to model multi-agent system problems such as consensus, formation, etc. In fact, the problems of the multi-agent systems, such as formation, can be considered a consensus problem. Consensus simply means that values of a quantity of all agents in a system should be converged to a same value. This value can be speed of agents, position of agents, etc. If consensus is reached, then $\left(L \otimes I_{m}\right) r=0$ by remembering that Laplacian matrix always has a zero eigenvalue with 1 vector as the right eigenvector. $m$ is the quantity dimension of $r$, obviously. Also, if $\left(L \otimes I_{m}\right) r=0$ is satisfied, then it will mean that consensus is accomplished due to:

$$
\begin{aligned}
\left(L \otimes I_{m}\right) r & =0 \Rightarrow r \in \operatorname{span}\left(1 \otimes r_{c}\right), r_{c} \in \mathbb{R}^{3}\left(L \otimes I_{m}\right) r \\
& =0 \Rightarrow r \in \operatorname{span}\left(1 \otimes r_{c}\right), r_{c} \in \mathbb{R}^{3}
\end{aligned}
$$

where, $r_{c}$ is consensus value and depends on initial value of $r$. Now, by substituting $r$ with $\delta=r-r_{\text {form }}$ in which $r_{\text {form }}$ is reference vector of the desired formation, one can write the following equation:

$$
\begin{aligned}
\left(L \otimes I_{m}\right) \delta=0 & \Rightarrow \delta \in \operatorname{span}\left(1 \otimes r_{e}\right), r_{e} \in \mathbb{R}^{3} \\
& \Rightarrow r \in \operatorname{span}\left(1 \otimes r_{e}\right)+r_{f} .
\end{aligned}
$$

It means that formation is reached because summing formation reference vector with a vector does not affect formation as shown in Figure 1. So if a decentralized control law causes $\left(L \otimes I_{m}\right) \delta=0$, then the desired formation will be achieved.

\section{Lemma [15]}

If a digraph has a spanning tree and the associated Laplacian graph is $L$, then there exists a symmetric positive definite matrix $P$ satisfying the following equation:

$$
P L+L^{T} P=Q
$$
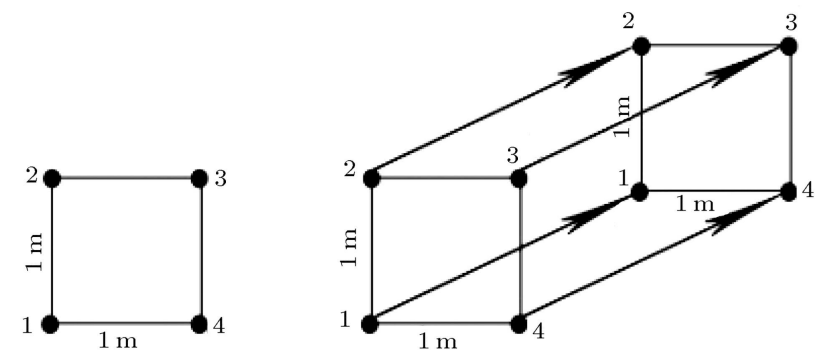

Figure 1. Summing formation reference vector of a square formation $\left(\left[\begin{array}{lllllllllll}0 & 0 & 1 & 0 & 1 & 1 & 1 & 1 & 1 & 1 & 0\end{array}\right]^{T}\right.$ ) with a vector that does not affect formation. 
where, $Q$ is a positive semi-definite matrix. This lemma has been proven in [15] using Theorems 4.6 and 4.29 from [16,17], respectively.

\section{Designing decentralized controller via backstepping}

Characteristics of the network of the system can affect the control law design. So, at first step of the controller design, general characteristics of the networks considered in the present work should be defined. After that, decentralized control laws are designed to track the velocity bounded trajectories by formation of integrator and double-integrator agents. Robust controller design is the final step of the controller design.

\subsection{General properties of network}

Narrow necessary total bandwidth to perform missions always is preferred. Decreasing this bandwidth even in case of the decentralized controllers is recommended because it results in ad-hoc communications with more narrow bandwidths. Therefore, using decentralized controllers on digraph networks is preferred to their usage in undirected graphs, which needs higher total bandwidth. So, the chosen proximity graph of this study is a digraph. In leader-follower approach, the desired trajectory of mission is sent from a station to the leader of the multi-agent system (in case of missions which are not predefined) or is set on the leader, previously, while followers communicate together and with the leader. In the mission of this study, it is assumed that the leader does not receive any information from other agents to reduce the required bandwidth. The digraph of this study has spanning tree with the leader as the root of the tree. Consequently, the general properties of the network of this study can be listed as follows:

- The proximity graph of the network is a directed graph;

- No follower sends information to the leader;

- The trajectory which should be tracked by the formation is sent only to the leader from the central station or is defined, previously, only for the leader;

- The digraph of the network has a spanning tree with the root located on the leader.

\subsection{Controller design for network of integrator agents}

For an Integrator agent, equation of motion is $\dot{r}_{i}=u_{i}$, obviously. Therefore, proper control input of the leader agent can be adopted as $u_{L}=\dot{r}_{d}-K_{L} e_{r_{L}}$ in which $K_{L}$ is a positive constant and $r_{d}$ is the desired value of $r_{L}$ and $e_{r_{L}}=r_{L}-r_{d}$. This form of input makes the tracking error for the leader exponentially stable with zero equilibrium point, obviously. To design the decentralized controller of the system, a Lyapunov function, such as $V_{f}=\frac{1}{2} \delta^{T}\left(L^{T} P L \otimes I_{m}\right) \delta$, can be considered, in which matrix $P$ is the matrix defined in the lemma. So, derivative of this Lyapunov function will be:

$$
\begin{aligned}
\dot{V}_{f}= & \frac{1}{2}\left(U^{T}\left(L^{T} P L \otimes I_{m}\right) \delta\right. \\
& \left.+\delta^{T}\left(L^{T} P L \otimes I_{m}\right) U\right) .
\end{aligned}
$$

$U$ is the input vector of all the agents in the recent equation. Without losing generality, it can be assumed that leader agent is the first agent. By choosing $U$ as:

$$
U=-K_{0}\left(L \otimes I_{m}\right) \delta+\left[\begin{array}{c}
u_{L} \\
0_{(n-1) \times 1}
\end{array}\right]
$$

in which $k_{0}$ is a positive constant, it can be concluded that:

$$
\begin{aligned}
\dot{V}_{f} & \leq \frac{1}{2}\left(-k_{0} \delta^{T}\left(L^{T} Q L \otimes I_{m}\right) \delta+\delta^{T}\left(L^{T} Q^{\prime} L \otimes I_{m}\right) \delta\right) \\
& +\left\|\dot{r}_{d}\right\|^{2}+K_{L}^{2}\left\|e_{r_{L}}\right\|^{2} .
\end{aligned}
$$

The matrix $Q$ in the recent equation is the same as the $Q$ matrix in the lemma. In the derived inequality matrix, $Q^{\prime}=P L^{T} L P$, which is a positive semi-definite matrix due to the definition of the $P$ matrix. As explained previously, tracking error of the leader is exponentially stable with zero equilibrium point. So, a Lyapunov function for the leader tracking error can be found such that $\dot{V}_{L} \leq-K_{L}^{2}\left\|e_{r_{L}}\right\|^{2}$. Consequently, one can have the following inequality:

$$
\begin{aligned}
\dot{V}_{f} & +\dot{V}_{L} \leq \frac{1}{2}\left(-k_{0} \lambda_{2}(Q)+\lambda_{\max }\left(Q^{\prime}\right)\right)\left\|\left(L \otimes I_{m}\right) \delta\right\|^{2} \\
& +\left\|\dot{r}_{d}\right\|^{2} .
\end{aligned}
$$

$\lambda_{2}$ denotes the second smallest eigenvalue (the first one is zero) and $\lambda_{\max }$ is notation of the largest eigenvalue, definitely. If $k_{0} \geq \frac{\lambda_{\max }\left(Q^{\prime}\right)}{\lambda_{2}(Q)}$, then it can be concluded that if the velocity of the trajectory is bounded then formation keeping error will be upbounded with $\frac{2 \max \left(\left\|\dot{r}_{d}\right\|\right)^{2}}{\left(k_{0} \lambda_{2}(Q)-\lambda_{\max }\left(Q^{\prime}\right)\right)}$. Therefore, increasing $k_{0}$ can increase formation precision. Notice that input is fully decentralized for the followers and fully centralized for the leader because the leader does not listen to the followers and the first row of $L$ is zero, accordingly.

\subsection{Controller design for network of double integrator agents}

For double integrator agents, equation of motion is $\ddot{r}_{i}=u_{i}$, apparently. To perform Backstepping control design, a Lyapunov function should be candidate. 
The candidate function is the same as the candidate function of the integrator agents, but the derivative of this Lyapunov function for double integrator agents will be:

$$
\begin{aligned}
\dot{V}_{f}= & \frac{1}{2}\left(\dot{r}^{T}\left(L^{T} P L \otimes I_{m}\right) \delta\right. \\
& \left.+\delta^{T}\left(L^{T} P L \otimes I_{m}\right) \dot{r}\right) .
\end{aligned}
$$

By defining $Z=\dot{r}-\dot{r}_{s}$ in which $\dot{r}_{s}$ is defined as:

$$
\begin{aligned}
& \dot{r}_{s}=-K_{0}\left(L \otimes I_{m}\right) \delta+\left[\begin{array}{c}
\dot{r}_{s_{L}} \\
0 m(n-1) \times 1
\end{array}\right], \\
& \dot{r}_{s_{L}}=\dot{r}_{d}-k_{L} e_{r_{L}} .
\end{aligned}
$$

Eq. (8) will result in the following inequality:

$$
\begin{aligned}
\dot{V}_{f} & \leq \frac{1}{2}\left(Z^{T} Z\right)+\frac{1}{2}\left(-k_{0} \delta^{T}\left(L^{T} Q L \otimes I_{m}\right) \delta\right. \\
& \left.+2 \delta^{T}\left(L^{T} Q^{\prime} L \otimes I_{m}\right) \delta\right)+\frac{\left\|\dot{r}_{s_{L}}\right\|^{2}}{2} .
\end{aligned}
$$

Now, if $\dot{Z}=-K_{Z} Z$ in which $K_{Z}$ is a positive constant, then $Z$ will be exponentially stable with zero equilibrium point. Therefore, a Lyapunov function $V_{Z}$ can be found such as $\dot{V}_{Z} \leq-\frac{1}{2} Z^{T} Z$. Consequently, the following inequality is correct due to definition of $\dot{r}_{s_{L}}$ :

$$
\begin{aligned}
\dot{V}_{f} & +\dot{V}_{Z} \leq \frac{1}{2}\left(-k_{0} \delta^{T}\left(L^{T} Q L \otimes I_{m}\right) \delta\right. \\
& \left.+2 \delta^{T}\left(L^{T} Q^{\prime} L \otimes I_{m}\right) \delta\right)+\left\|\dot{r}_{d}\right\|^{2} \\
& +K_{L}^{2}\left\|e_{r_{L}}\right\|^{2} .
\end{aligned}
$$

$\dot{Z}=-K_{Z} Z$ will result in control input vector as:

$$
\begin{aligned}
U= & -k_{0}\left(L \otimes I_{m}\right) \dot{r}-K_{Z}\left(\dot{r}+k_{0}\left(L \otimes I_{m}\right) \delta\right) \\
& +\left[\begin{array}{c}
\ddot{r}_{d}-K_{L} \dot{e}_{r_{L}}+K_{Z}\left(\dot{r}_{d}-K_{L} e_{r_{L}}\right) \\
0_{m(n-1) \times 1}
\end{array}\right],
\end{aligned}
$$

which is fully decentralized for the followers and fully centralized for the leader because the first row of the $L$ is zero. By Eq. (12), input of the leader will be $u_{L}=\ddot{r}_{d}-\left(K_{L}+K_{Z}\right) \dot{e}_{r_{L}}-K_{Z} K_{L} e_{r_{L}}$. This form of the control input of the leader makes trajectory tracking error of the leader exponentially stable with zero equilibrium point. So, a Lyapunov function for the leader can be found such that $\dot{V}_{L} \leq-K_{L}\left\|e_{r_{L}}\right\|^{2}$. Then, Inequality (11) will be changed to:

$$
\begin{aligned}
\dot{V}_{f} & +\dot{V}_{Z}+\dot{V}_{L} \leq \frac{1}{2}\left(-k_{0} \lambda_{2}(Q)\right. \\
& \left.+2 \lambda_{\max }\left(Q^{\prime}\right)\right)\left\|\left(L \otimes I_{m}\right) \delta\right\|^{2}+\left\|\dot{r}_{d}\right\|^{2} .
\end{aligned}
$$

If one chooses $k_{0}$ such as $k_{0} \geq \frac{2 \lambda_{\max }\left(Q^{\prime}\right)}{\lambda_{2}(Q)}$ and velocity of the trajectory is bounded, then $\left\|\left(L \otimes I_{m}\right) \delta\right\|^{2}$ is up-bounded with $\frac{2 \max \left(\left\|\dot{r}_{d}\right\|\right)^{2}}{\left(k_{0} \lambda_{2}(Q)-2 \lambda_{\max }\left(Q^{\prime}\right)\right)}$. The error of the formation, while tracking the trajectory, can be reduced by increasing the gain $k_{0}$ as possible.

\subsection{Robustness of formation under changing network topologies}

Because of terms, conditions, and changes of surrounded environment, topology of networks during missions may be altered accidentally. So, robustness of multi-agent systems against the variations of the proximity graph of the system is too important. Therefore, robustness of the derived control law in the previous section should be guaranteed.

For a network of agents with the mentioned characteristics, finite forms of proximity graphs and associated unweighted Laplacian matrices can be assigned. Moreover, Laplacian matrices of some of them have the same eigenvalues and eigenvectors and so they are the same from controller design point of view. Consider that there are $m$ possible independent proximity graphs with the explained characteristics on a network of agents denoted by $\Gamma=\left\{L_{j} \mid j=1,2, \cdots, m\right\}$. For each member of $\Gamma$ with a constant $Q_{1}$, matrices $P_{j}, Q_{j}$, and $Q_{j}^{\prime}$ are calculated. The second smallest eigenvalues of all $Q_{j}$ s, denoted by $\lambda_{2}\left(Q_{j}\right)$, and the largest eigenvalue of $Q_{j}^{\prime}$, denoted by $\lambda_{n}\left(Q_{j}^{\prime}\right)$, are derived accordingly. So, for the system of integrator or double integrators, sets $\Lambda_{1}$ and $\Lambda_{2}$ can be found, respectively, as:

$$
\begin{aligned}
& \Lambda_{1}=\left\{\frac{\lambda_{\max }\left(Q_{j}^{\prime}\right)}{\lambda_{2}\left(Q_{j}\right)} \mid j=1,2, \cdots, m\right\}, \\
& \Lambda_{2}=\left\{\frac{2 \lambda_{\max }\left(Q_{j}^{\prime}\right)}{\lambda_{2}\left(Q_{j}\right)} \mid j=1,2, \cdots, m\right\} .
\end{aligned}
$$

So, to design robust formation controller under varying topology networks, it is sufficient that $k_{0} \geq \max (\Lambda)$.

\section{Simulation results}

In this section, some simulations have been performed on a multi-agent system of four double integrator agents. The desired shape of the formation is the square of Figure 1 and reference vector of formation is the same, too. At first, effect of $k_{0}$ on the precision of the formation keeping while tracking trajectory of a path with bounded velocity is examined. So, in the next simulation, it is shown that the designed controller loses its capability in keeping formation while tracking trajectories with boundless velocities. In the other words, followers cannot be located on the formation shape around the leader while the leader tracks its desired trajectory. The last simulation is around the robustness capabilities of the designed control law 
against the changes of the Laplacian matrix of the network and it is verified that the proposed controller exhibits enough robustness when it is subjected to the aforementioned surrounded environmental conditions which cause changes in the topology of the network. In all these three simulations, it is assumed that all agents start to move from stationary situation and their initial positions are:

$$
\begin{aligned}
r_{0} & =\left[\begin{array}{llllllll}
r_{0_{1}} & r_{0_{2}} & r_{0_{3}} & r_{0_{4}}
\end{array}\right]^{T} \\
& =\left[\begin{array}{llllllll}
2 & 3 & 4 & 7 & 6 & 2 & 1 & 6
\end{array}\right]^{T} .
\end{aligned}
$$

\subsection{Effect of $k_{0}$ value on precision of formation}

In this simulation, it is verified that increasing $k_{0}$ will reduce the error of the formation, as claimed. Desired trajectory of leader is defined by a velocity bounded trajectory as $r_{d}=[t \sin (t)]^{T}$. Therefore, the designed control law can keep formation precision while tracking the trajectory. The proximity graph of the network is assumed to be a chain graph like the left side graph in Figure 2 having all the considered characteristics. By considering $Q_{1}$ of lemma as $(0.01) I_{3 \times 3}$, one can obtain $\lambda_{2}(Q)=0.0034, \lambda_{n}\left(Q^{\prime}\right)=0.0037$. Therefore, from the condition found while backstepping, $k_{0}$ should be adopted such that $k_{0} \geq 2.13$. Consequently, for the first simulation, $k_{0}$ and all gains are set to 5 . Values of other gains are assumed 5, too. In Figure 3, $X$ and $Y$ coordinates of agents during mission have been
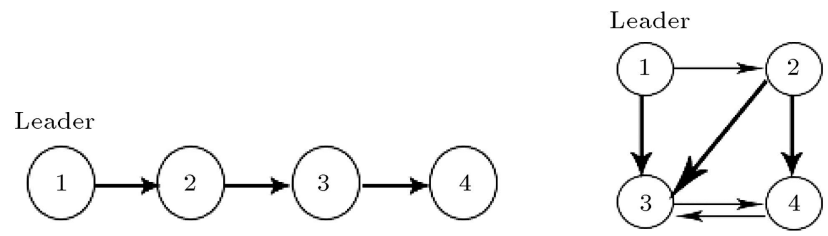

Figure 2. Proximity graphs of network in simulations.

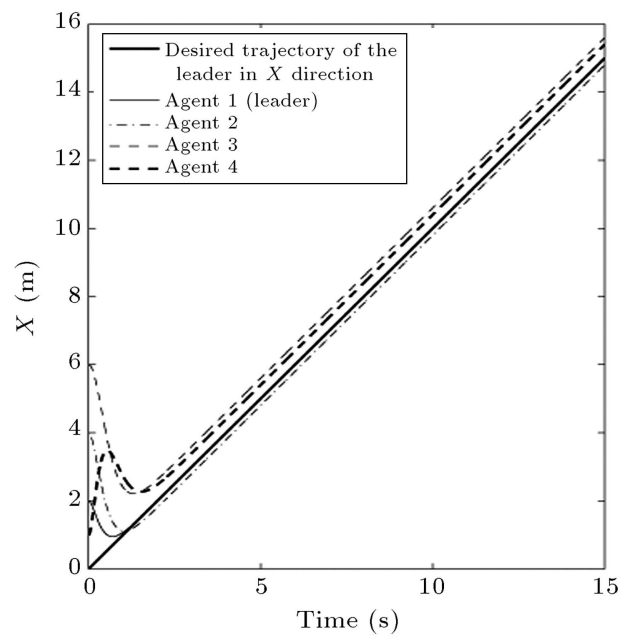

demonstrated and it is obvious that the formation has been kept with a bounded error, as claimed formerly. From Figure 4, it is obvious that velocities of agents in $X$ direction have converged to a same value because the acceleration of the desired trajectory in $X$ direction is zero. As depicted in Figure 4, consensus on velocity in $Y$ direction has a bounded error because acceleration in $Y$ direction is bounded. Also, in Figure $5,\left\|\left(L \otimes I_{2}\right) \delta\right\|$ as formation keeping error is plotted versus time and it has been verified that error of formation demonstrated by a thick line is under the up-bound derived in backstepping. Moreover, Figure 5 demonstrates that the shape of the formation is too different from the desired square shape.

In the next simulation, only $k_{0}$ is changed to 20 to verify that if the formation precision is improved or not. Plot of $\left\|\left(L \otimes I_{2}\right) \delta\right\|$ versus time depicts degradation of formation error and its bound in comparison with previous simulation and the square formation has been achieved well as depicted in Figure 6. Therefore, as proved, higher $k_{0}$ values increase precision of formation and decrease error of formation. Of course, increasing $k_{0}$ in real applications may cause saturation in actuators of robots.

\subsection{Controller failure in tracking trajectories with unbound velocities}

In this simulation, the desired trajectory of leader is assumed to be $r_{d}=\left[\begin{array}{ll}t^{2} & \sin (t)\end{array}\right]^{T}$. Since $\dot{r}_{d}=$ $[2 t \cos (t)]^{T}$, the velocity of the desired trajectory is unbounded. So, the formation error will be unbounded by the designed controller. The proximity graph of the system and $Q_{1}$ matrix are the same as those in previous simulations. The gains of the system by considering the condition derived in Backstepping are set to 5 . In Figure $7, X$ and $Y$ coordinates of the agents during mission are demonstrated. As demonstrated in Figure 7 and proved in Backstepping,

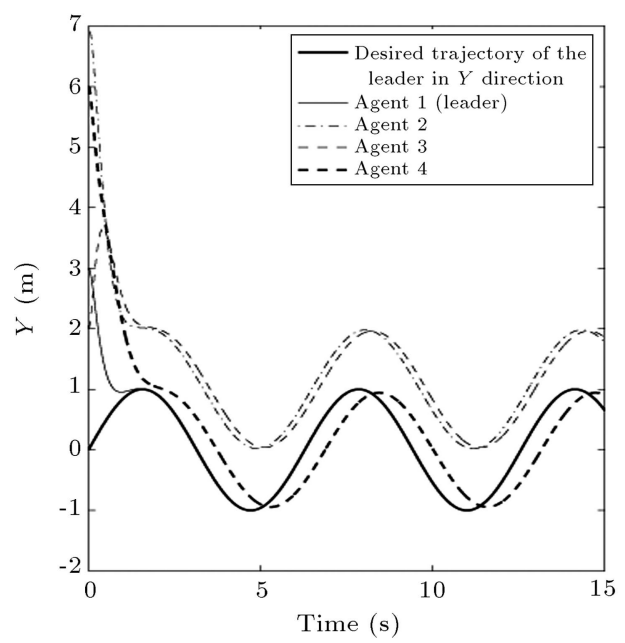

Figure 3. Coordinates of agents during mission in the first simulation. 

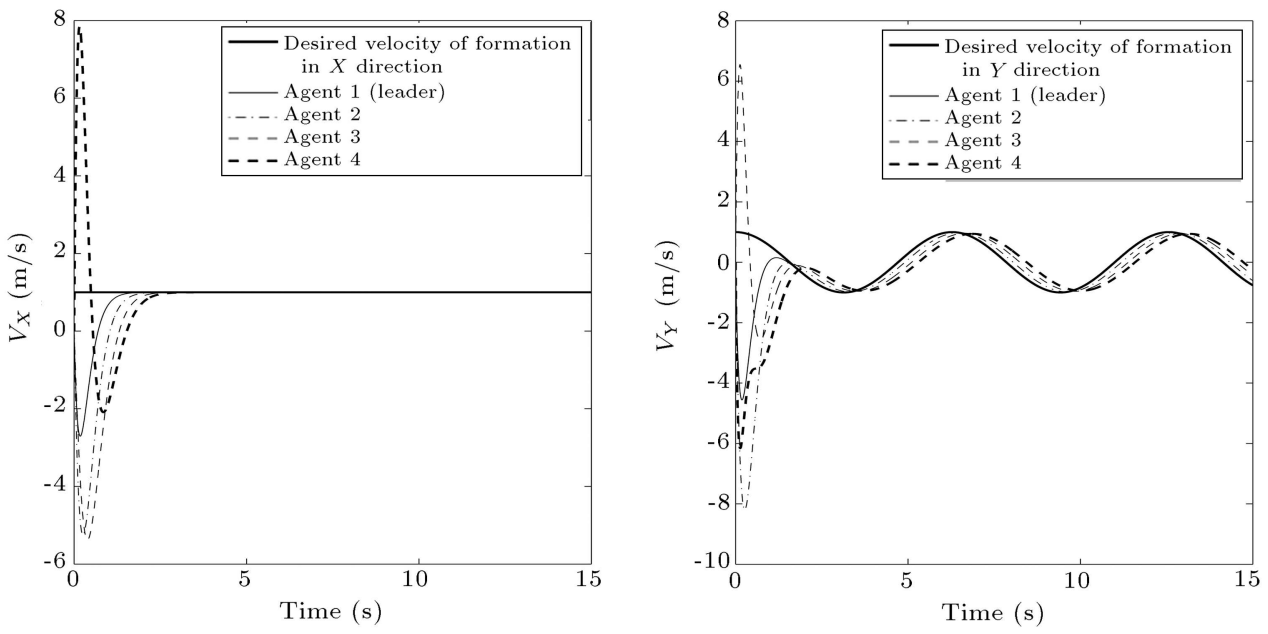

Figure 4. Velocity of agents in $X$ direction during mission in the first simulation.

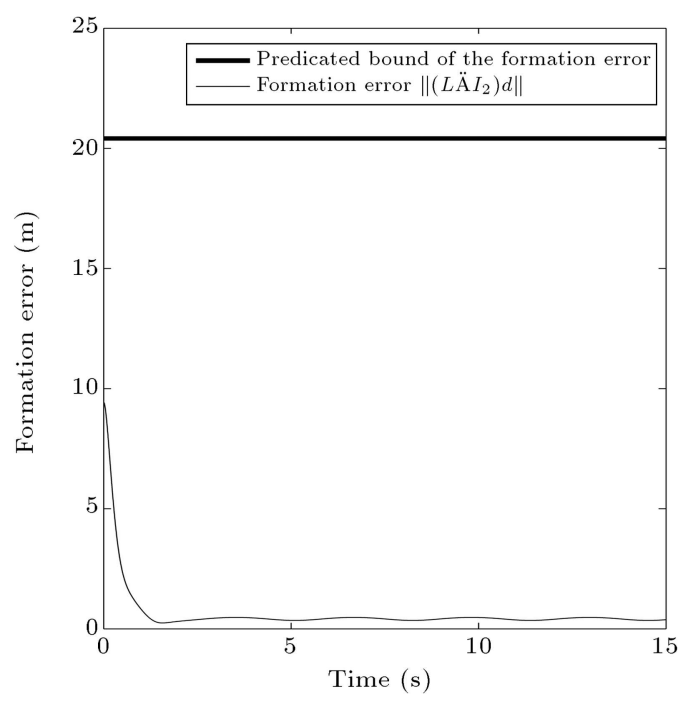

(a)

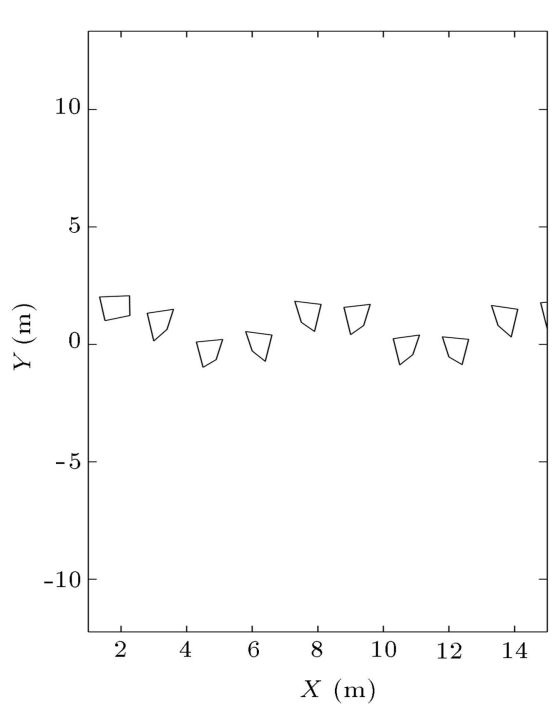

(b)

Figure 5. Error of formation and bound of error (a) and shape of formation during mission for $k_{0}=5$ (b).

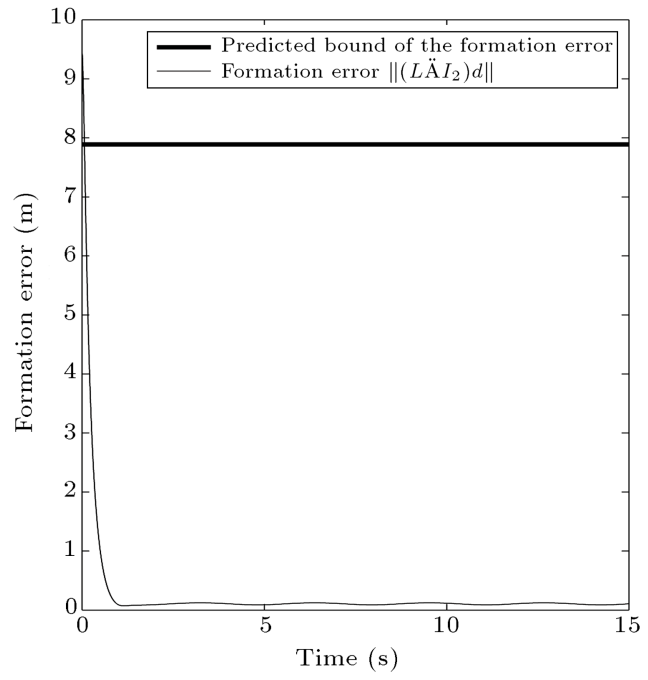

(a)

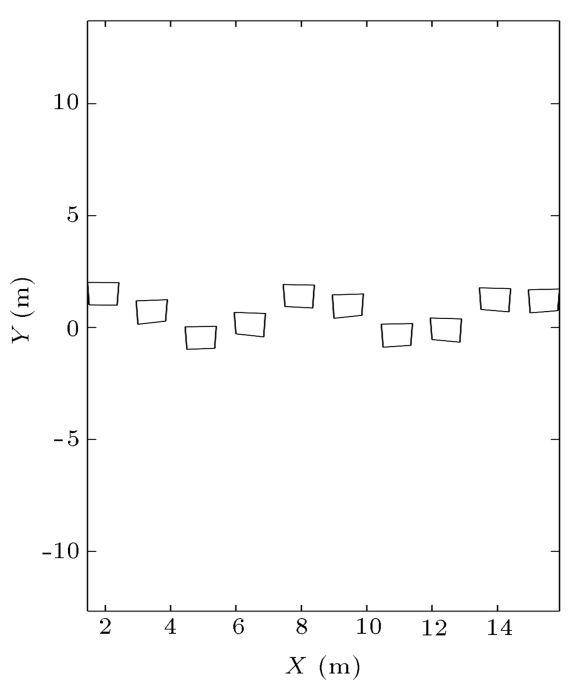

(b)

Figure 6. Error of formation and bound of error (a) and shape of formation during mission for $k_{0}=20$ (b). 

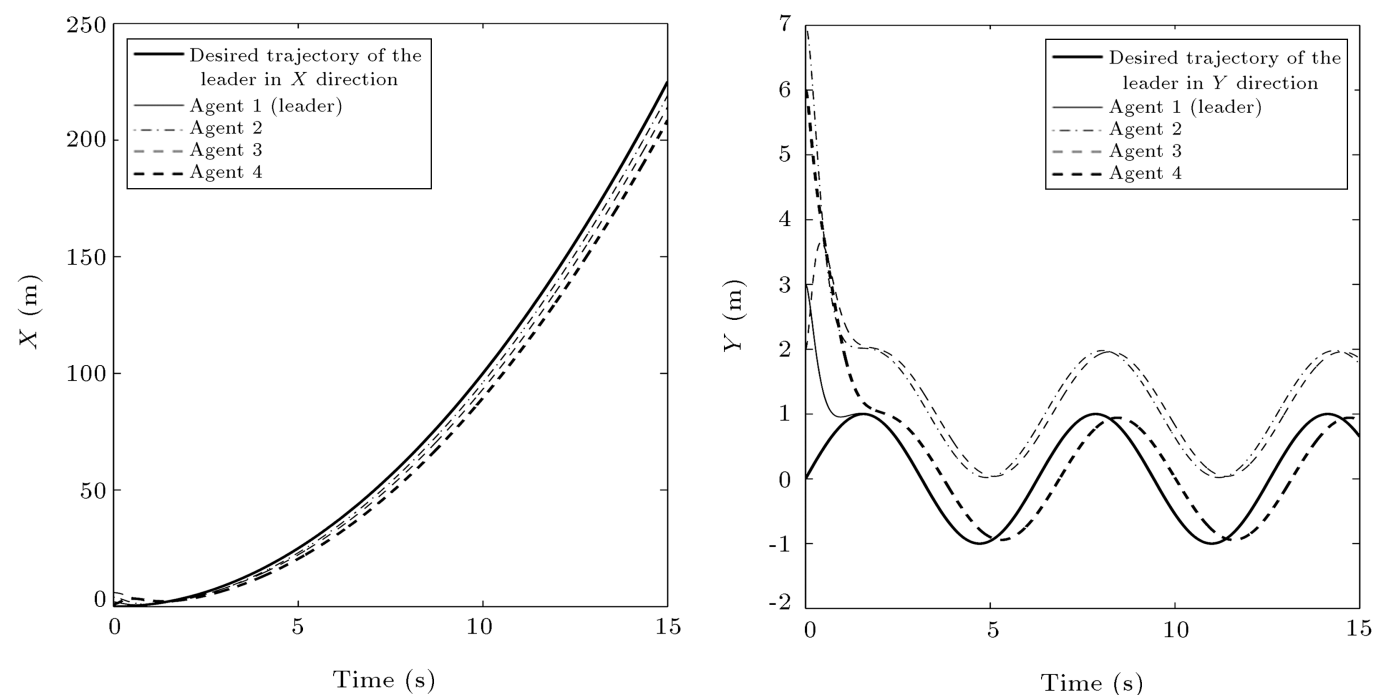

Figure 7. Coordinates of agents during mission in case of tracking trajectory with unbounded velocity.

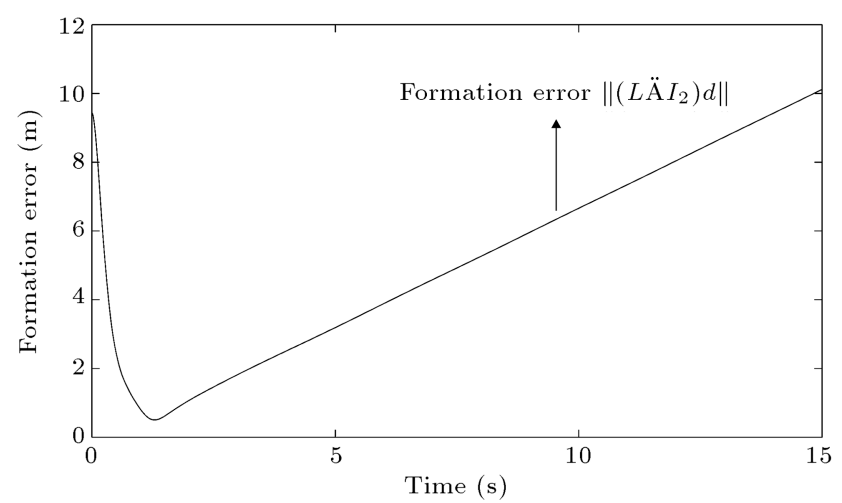

Figure 8. Error of formation during mission in case of tracking trajectory with unbounded velocity.

the error of the formation in $X$ direction increases during the mission because the velocity of the desired trajectory in $X$ direction increases by time. Nevertheless, the formation along $Y$ axis has bounded error as proven and depicted in Figure 7 . So, as proven formerly, $\left\|\left(L \otimes I_{2}\right) \delta\right\|$ would increase and the error of the formation would not be bounded, as depicted in Figure 8. Consequently, in case of the trajectories with unbounded velocities, the designed controller lost its capability.

\subsection{Robust formation control against network topology changes}

In this simulation, a formation controller, which is robust against the network topology changes, has been designed for a leader-follower multi-agent system of four double-integrator agents. A Leader-follower multi-agent system with four agents has 144 independent forms of unweighted Laplacian matrices. Set $\Lambda_{2}$ is calculated by the $Q_{1}$ matrix of the previous simulations and its maximum is 34.08. Therefore, if $k_{0} \geq 34.08$, then the controller will be robust against the changes of the proximity graph of the network. Two simulations have been performed to analyze effect of $k_{0}$ value on the robustness of the formation against the changes of proximity graphs. In one of the simulations, $k_{0}<34.08$ and in another, $k_{0} \geq 34.08$, while other gains are set to 5 . In both of the simulations, the simulation starts with proximity graph and unweighted Laplacian matrix of left graph of Figure 2 and at $t=5.3$ seconds, the proximity graph changes to right graph of this figure, suddenly. The desired trajectory of formation in both of the simulations is same as that of the first simulation. In Figure 9, the error of the formation during the mission for $k_{0}=20$ and $k_{0}=40$ is demonstrated. Comparing these two plots represents that the deflection of the formation from its steady state value in the case of $k_{0}=40$ is less than when $k_{0}=20$, as predicted.

\section{Conclusion}

This paper introduces a robust decentralized control law to guide formations of special category of leaderfollower multi-agent systems on bounded velocity trajectories which are not predefined or only defined for the leader agent. By implementing the proposed control laws, formation keeping will have bounded error while the desired trajectory is tracked by leader. This bound can be decreased by increasing a gain of the controller. Control laws are redesigned to be robust against the proximity graph changes. Simulations verified the capabilities of the designed control laws and their robustness, appropriately. Designing observer for agents to estimate the velocity of the trajectory may decrease the formation error. Moreover, using this control law, which enters the saturation problem, in real networks is recommended. 

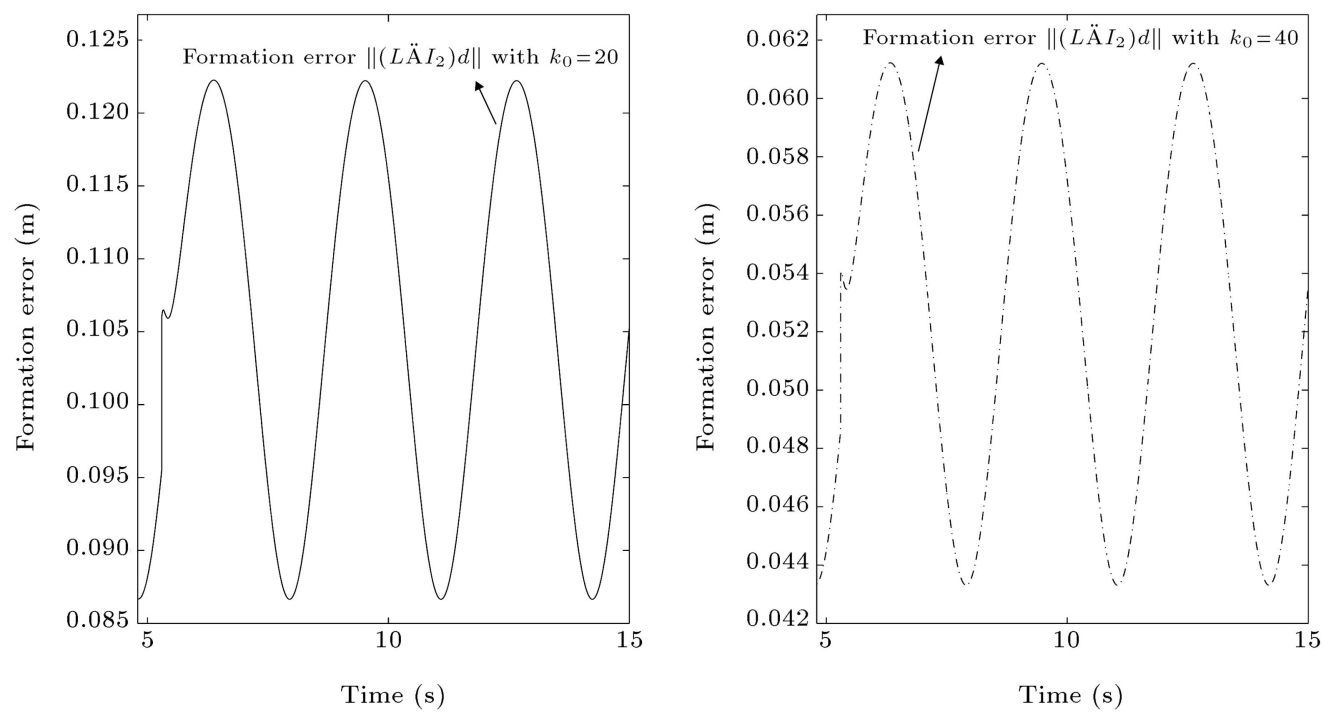

Figure 9. Error of formation with uncertain network topology during mission for $k_{0}=20$ (a) and $k_{0}=40$ (b); less deviation around $t=5.3 \mathrm{~s}$ is obvious when $k_{0}=40$.

\section{References}

1. Wang, H. "Flocking of networked uncertain EulerLagrange systems on directed graphs", Automatica, 49, pp. 2774-2779 (2013).

2. Zhai, C. and Hong, Y. "Decentralized sweep coverage algorithm for multi-agent systems with workload uncertainties", Automatica, 49, pp. 2154-2159 (2013).

3. Su, H., Wang, X. and Chen, G. "Rendezvous of multiple mobile agents with preserved network connectivity", Systems \& Control Letters, 59, pp. 313-322 (2010).

4. Meurer, T. and Krstic, M. "Finite-time multi-agent deployment: A nonlinear PDE motion planning approach", Automatica, 47, pp. 2534-2542 (2011).

5. Lawton, J.R.T., Beard, R.W. and Young, B.J. "A decentralized approach to formation maneuvers", Robotics and Automation, IEEE Transactions on, 19, pp. 933-941 (2003).

6. Sayyaadi, H. and Doostmohammadian, M.R. "Finitetime consensus in directed switching network topologies and time-delayed communications", Scientia Iranica, 18, pp. 75-85 (2011).

7. Zhang, W., Wang, Z. and Guo, Y. "Backsteppingbased synchronisation of uncertain networked Lagrangian systems", International Journal of Systems Science, 45, pp. 145-158 (2012).

8. Balch, T. and Arkin, R.C. "Behavior-based formation control for multirobot teams", Robotics and Automation, IEEE Transactions on, 14, pp. 926-939 (1998).

9. Mehrjerdi, H., Ghommam, J. and Saad, M. "Nonlinear coordination control for a group of mobile robots using a virtual structure", Mechatronics, 21, pp. 1147-1155 (2011).

10. Consolini, L., Morbidi, F., Prattichizzo, D. and
Tosques, M. "Leader-follower formation control of nonholonomic mobile robots with input constraints", Automatica, 44, pp. 1343-1349 (2008).

11. Liang, H., Sun, Z. and Wang, J. "Robust decentralized attitude control of spacecraft formations under timevarying topologies, model uncertainties and disturbances", Acta Astronautica, 81, pp. 445-455 (2012).

12. Tanner, H.G., Pappas, G.J. and Kumar, V. "Leaderto-formation stability", Robotics and Automation, IEEE Transactions on, 20, pp. 443-455 (2004).

13. Popov, A. and Werner, H. "Robust stability of a multi-agent system under arbitrary and time-varying communication topologies and communication delays", Automatic Control, IEEE Transactions on, 57, pp. 2343-2347 (2012).

14. Ren, W. and Beard, R., Distributed Consensus in Multi-vehicle Cooperative Control Theory and Applications, London, Springer-Verlag (2008).

15. Wenlin, Z., Zheng, W. and Yi, G. "Robust consensus for uncertain multi-agent systems on directed communication topologies", In Decision and Control (CDC), 49th IEEE Conference on, pp. 6317-6322 (2010).

16. Khalil, H.K., Nonlinear Systems, Upper Saddle River, New Jersey, Prentice Hall (2002).

17. Qu, Z., Cooperative Control of Dynamical Systems, Application to Autonomous Vehicles, London, Springer-Verlag (2009).

\section{Biographies}

Hassan Sayyaadi received his BS degree from Amirkabir University of Technology, Tehran, Iran, in 1987, then, he received his MS degree from Sharif University of Technology, Tehran, Iran, in 1990 and he received his $\mathrm{PhD}$ degree from the University of Tokyo, Japan, in 2001, all in Mechanical Engineering. From 1990 
to 1996, he was with the Mechanical Engineering Department of Sharif University of Technology and from 2001, after his PhD studies, he has again been with the same department and university. Now, he is an Associate Professor and his research interests include dynamics and control, robotics and mechanisms, artificial intelligence, and neural networks.
Ali Soltani received his BS and MS degrees from Sharif University of Technology, Tehran, Iran, in 2007 and 2010, respectively and he started PhD course in the same university in 2011, all in Mechanical Engineering. Now, he is $\mathrm{PhD}$ candidate student and progressing his $\mathrm{PhD}$ thesis. His research is all about multi-agent robotic systems and quadrotors. 